Also available at http://amc.imfm.si

ISSN 1855-3966 (printed edn.), ISSN 1855-3974 (electronic edn.)

ARS MATHEMATICA CONTEMPORANEA 5 (2012) 289-293

\title{
Facial parity edge coloring of outerplane graphs
}

\author{
Július Czap \\ Department of Applied Mathematics and Business Informatics, Faculty of Economics, \\ Technical University of Košice, Němcovej 32, 04001 Košice, Slovakia
}

Received 6 September 2011, accepted 1 December 2011, published online 28 March 2012

\begin{abstract}
A facial parity edge coloring of a 2-edge-connected plane graph is such an edge coloring in which no two face-adjacent edges (consecutive edges of a facial walk of some face) receive the same color, in addition, for each face $f$ and each color $c$, either no edge or an odd number of edges incident with $f$ is colored with $c$. It is known that any 2-edgeconnected plane graph has a facial parity edge coloring with at most 92 colors. In this paper we prove that any 2-edge-connected outerplane graph has a facial parity edge coloring with at most 15 colors. If a 2-edge-connected outerplane graph does not contain any inner edge, then 10 colors are sufficient. Moreover, this bound is tight.
\end{abstract}

Keywords: Plane graph, facial walk, edge coloring.

Math. Subj. Class.: 05C10, 05C15

\section{Introduction}

The facial parity edge coloring concept was introduced in [4]. The motivation has come from the papers of Bunde et al. [1,2]. They introduced parity edge colorings of graphs. A parity walk in an edge coloring of a simple graph is a walk along which each color is used an even number of times. Let $p(G)$ be the minimum number of colors in an edge coloring of $G$ having no parity path (parity edge coloring). Let $\widehat{p}(G)$ be the minimum number of colors in an edge coloring of $G$ in which every parity walk is closed (strong parity edge coloring). Clearly, every parity edge coloring is a proper edge coloring. Although there are graphs $G$ with $\widehat{p}(G)>p(G)[1]$, it remains unknown how large $\widehat{p}(G)$ can be when $p(G)=k$. In [1] it is mentioned that computing $p(G)$ or $\widehat{p}(G)$ is NP-hard even when $G$ is a tree.

The facial parity edge coloring can be considered as a relaxation of the parity edge coloring. We focus on facial cycles of plane graphs. This coloring has to satisfy the following two conditions:

E-mail address: julius.czap@tuke.sk (Július Czap) 
1. face-adjacent edges receive different colors,

2. for every color $c$ and every face $f$ the total number of occurrences of edges colored with $c$ on a facial walk of $f$ is odd or zero.

The authors of [4] proved that every 2-edge-connected plane graph has a facial parity edge coloring with at most 92 colors.

In this paper we substantially improve this bound for the class of 2-edge-connected outerplane graphs.

Note that the vertex version of this problem was investigated in [5]. The authors proved that every 2-connected plane graph admits a parity vertex coloring using at most 118 colors. Kaiser et al. [8] improved this bound to 97. Czap [3] proved that any 2-connected outerplane graph has such a coloring with at most 12 colors. The generalization of the parity coloring for graphs and set systems can be found in [6].

\section{Notation}

Let us introduce the notation used in this paper. A graph which can be embedded in the plane is called planar graph; a fixed embedding of a planar graph is called plane graph. Outerplane graphs are plane graphs such that every vertex lies on the outer face.

A bridge is an edge whose removal increases the number of components. A graph which contains no bridge is said to be bridgeless or 2-edge-connected. In this paper we consider connected bridgeless plane graphs, multiple edges and loops are allowed.

Given a graph $G$ and one of its edges $e=u v$ (the vertices $u$ and $v$ do not have to be different), the contraction of $e$ consists of replacing $u$ and $v$ by a new vertex adjacent to all the former neighbors of $u$ and $v$, and removing the loop corresponding to the edge $e$. (We keep multiple edges if they arise.)

Two (distinct) edges are face-adjacent if they are consecutive edges of a facial walk of some face $f$.

A $k$-edge coloring of a graph $G=(V, E)$ is a mapping $\varphi: E(G) \rightarrow\{1, \ldots, k\}$. We say that an edge coloring of a plane graph $G$ is facially proper if no two face-adjacent edges of $G$ receive the same color. The facial parity edge coloring of a 2-edge-connected plane graph is a facially proper edge coloring such that for each face $f$ and each color $c$, either no edge or an odd number of edges incident with $f$ is colored with $c$.

Question 2.1. What is the minimum number of colors $\chi_{p}^{\prime}(G)$ such that a 2-edge-connected plane graph $G$ has a facial parity edge coloring with at most $\chi_{p}^{\prime}(G)$ colors?

\section{Results}

Lemma 3.1. Let $C_{n}$ be a cycle on $n$ edges, $n \geq 1$. Then $\chi_{p}^{\prime}\left(C_{n}\right) \leq 5$.

Proof. If $n=1$, then we use one color. Let $n=4 k+z$, where $k$ is a non-negative integer and $z \in\{2,3,4,5\}$. We repeat $k$ times the pattern $1,2,1,2$ and then use colors $1,2, \ldots, z$. The colors 1 and 2 are thus used $2 k+1$ times, the remaining three colors are used at most once.

An edge of a plane graph not incident with the outer face is called inner edge.

Theorem 3.2. Let G be a 2-edge-connected outerplane graph with no inner edge (bridgeless cactus graph). Then $\chi_{p}^{\prime}(G) \leq 10$. Moreover, this bound is tight. 
Proof. First we prove that the edges of $G$ can be colored with at most 5 colors, say $1,2,3,4,5$, in such a way that for every inner face $f$ and every color $c \in\{1,2,3,4,5\}$, either no edge or an odd number of edges incident with $f$ is colored with $c$, in addition, face-adjacent edges receive different colors.

The proof is by induction on the number of inner faces. If $G$ has one inner face, then the statement follows from Lemma 3.1. Let $G$ have $k$ inner faces $f_{1}, \ldots, f_{k}$ and assume that the face $f_{k}$ is such a face of $G$ that the corresponding vertex in a block graph of $G$ is a leaf. Let $H$ be a subgraph of $G$ consisting of faces $f_{1}, \ldots, f_{k-1}$. The graph $H$ has fewer inner faces than $G$, hence it has a required coloring. It is easy to extend the coloring of $H$ to a coloring of $G$. Assume that the boundary of $f_{k}$ is a cycle $C$. Clearly, $C$ and $H$ have exactly one vertex $v$ in common. There are at most two forbidden colors for the edges of $C$ incident with $v$. We have five colors, hence there is such a facial parity edge coloring of the cycle $C$ that no two face-adjacent edges incident with $v$ receive the same color in $G$.

In the next step we recolor some edges. Assume that a color $i \in\{1,2,3,4,5\}$ appears an even number of times in $G$. Let $f$ be an arbitrary inner face which is incident with an edge of color $i$. We recolor all the edges of color $i$ incident with $f$ with a new color $i+5$. Now the total number of occurrences of edges colored with $i$ and $i+5$ is odd in $G$.

This recoloring uses at most 10 colors.

To see that the upper bound is tight it is sufficient to consider the graph in Figure 1.

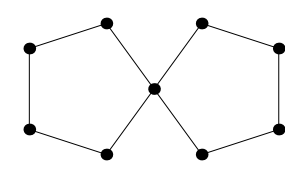

Figure 1: An example of a graph with no facial parity edge coloring using less than 10 colors.

Corollary 3.3. Let $G$ be a bridgeless cactus graph with no $C_{5}$. Then $\chi_{p}^{\prime}(G) \leq 8$. Moreover, this bound is tight.

Proof. First we show that among all cycles only the cycle on five edges requires 5 colors for a facial parity edge coloring. Let $n=4 k+z$, where $k$ is a non-negative integer and $z \in\{2,3,4,5\}$. If $z \neq 5$, then $\chi_{p}^{\prime}\left(C_{n}\right) \leq 4$ (see the proof of Lemma 3.1). If $n=4 k+5, k \geq 1$, then $\chi_{p}^{\prime}\left(C_{n}\right)=3$ (we repeat the pattern $1,2,3$ three times and then repeat $k-1$ times the pattern $1,2,1,2)$.

Now we can proceed as in the proof of Theorem 3.2.

Corollary 3.4. Let $G$ be a bridgeless cactus graph with no $C_{5}$ and no $C_{4 k}, k \geq 1$. Then $\chi_{p}^{\prime}(G) \leq 6$. Moreover, this bound is tight.

The dual $G^{*}$ of a plane graph $G$ can be obtained as follows: Corresponding to each face $f$ of $G$ there is a vertex $f^{*}$ of $G^{*}$, and corresponding to each edge $e$ of $G$ there is an edge $e^{*}$ of $G^{*}$; two vertices $f^{*}$ and $g^{*}$ are joined by the edge $e^{*}$ in $G^{*}$ if and only if their corresponding faces $f$ and $g$ are separated by the edge $e$ in $G$ (an edge separates the faces incident with it). The weak dual of a plane graph $G$ is the subgraph of the dual graph $G^{*}$ whose vertices correspond to the bounded faces of $G$. 
Lemma 3.5. [7] The weak dual of an outerplane graph is a forest.

We say that an edge coloring of a graph is odd, if each color class induces an odd subgraph (each vertex has an odd degree).

Lemma 3.6. Let $S_{n}$ be a star on $n$ edges, $n \geq 1$. Then it has a facially proper odd edge coloring using at most 5 colors.

Proof. We can use the coloring defined in the proof of Lemma 3.1.

Corollary 3.7. Let $T$ be a tree. Then it has a facially proper odd edge coloring using at most 5 colors.

Proof. Pick any vertex of $T$ to be the root. We color the edges of $T$ starting from the root to the leaves. In each step it is sufficient to find a facially proper odd edge coloring of a star with (at most) one precolored edge.

Corollary 3.8. Let $F$ be a forest. Then it has a facially proper odd edge coloring using at most 5 colors.

Theorem 3.9. Let $G$ be a 2-edge-connected outerplane graph. Then $\chi_{p}^{\prime}(G) \leq 15$.

Proof. First we color all the edges on the outer face with yellow color. The other edges let be green.

We successively contract the green edges and we obtain a graph $H$. The graph $H$ is outerplane with no inner edge, hence, from Theorem 3.2 it follows that there exists a facial parity edge coloring of $H$ with at most 10 colors.

In the following we extend the coloring of $H$ to a coloring of $G$. Let $F$ be the weak dual of $G$. Lemma 3.5 implies that $F$ is a forest. By Corollary 3.8, $F$ has a facially proper odd edge coloring which uses at most 5 colors. This coloring induces a coloring of the green edges of $G$ in a natural way. The coloring of the yellow edges with at most 10 colors and the coloring of the green edges with at most 5 colors (these five colors are different than the previous ten ones) induce a required coloring of $G$.

\section{Acknowledgments}

I would like to thank one of the anonymous referees for helpful comments and suggestions.

\section{References}

[1] D. P. Bunde, K. Milans, D. B. West and H. Wu, Parity and strong parity edge-coloring of graphs, Congressus Numerantium 187 (2007), 193-213.

[2] D. P. Bunde, K. Milans, D. B. West and H. Wu, Optimal strong parity edge-coloring of complete graphs, Combinatorica 28 (2008), 625-632.

[3] J. Czap, Parity vertex coloring of outerplane graphs, Discrete Math. 311 (2011), 2570-2573.

[4] J. Czap, S. Jendrol' and F. Kardoš, Facial parity edge colouring, Ars Math. Contemp. 4 (2011), 255-269.

[5] J. Czap, S. Jendrol' and M. Voigt, Parity vertex colouring of plane graphs, Discrete Math. 311 (2011), 512-520.

[6] J. Czap and Zs. Tuza, Partitions of graphs and set systems under parity constraints, preprint, 2011. 
[7] H. J. Fleischner, D. P. Geller and F. Harary, Outerplanar graphs and weak duals, J. Indian Math. Soc. 38 (1974), 215-219.

[8] T. Kaiser, O. Rucký, M. Stehlík and R. Škrekovski, Strong parity vertex coloring of plane graphs, IMFM Preprint series 49 (2011), \#1144. 